\title{
Resumen / Los lugares posibles de la creatividad
}

El presente trabajo es una reflexión y un análisis sobre los lugares de la creación particularmente en la ciencia. Específicamente en lo que hace a nuevos descubrimientos científicos en los diferentes contextos históricos y con el advenimiento de la modernidad en especial.

Luego el análisis se centra en la posmodernidad, con la particularidad de sus prácticas, y los posibles márgenes de la creación, particularmente la artística, en un contexto en el que la inmediatez, pero a la vez, el rechazo de lo real ligado a la tecnología y lo mediático, produce efectos que la diferencian de otros contextoshistóricos.

\section{Palabras clave}

arte - ciencia - creación - creatividad - crisis - cuerpo - método - modernidad - paradigma - posmodernidad razón - reglas- tiempo y espacio.

\section{Summary / Possible places of the creativity}

This work will be a reflexion and an analysis of the places use of the creation, specially those use for science. Specifically, in what makes the arrival of new scientific in discoveries different historical contexts and in special with the arrival of modernity.

Later, the analysis centres in the post-modernity, with the particularity of its practices, and in the possible margins of creation, specially artistic, in a context where immediately, though at the same time the rejection of reality bound to technology and to intervention, causes effects which differentiate it with other historical contexts.

\section{Key words}

art - body - creation - creativity - crisis- science - method - modernity - paradigm - pos-modernity - reason rules - time - space.

\section{Resumo / Os possíveis lugares da criatividade}

O seguinte trabalho representa uma reflexão e uma análise dos lugares de criação, particularmente na ciência.

Específicamente no que faz referência ao desenvolvimento de novos descobrimentos científicosocurridos nosdiferentes contextos históricos, e especialmente a chegada da modernidade.

A partir disso, osanálises centralizam-se na posmodernidade, com a particularidade de suas práticase seus possíveis marcos de criação, especialmente no meio artístico, em que a imediatez, e ao mesmo tempo a rejeição do real, vinculado a tecnología e ao mediático, produzem efeitos que diferenciam-se de outros contextos históricos.

\section{Palavras chave}

arte - ciência - creatividade - criação - corpo - espaço - método - modernidade - paradigma - posmodernidaderazão - regra - tempo.

\footnotetext{
* Centro de Estudios en Diseño y Comunicación, Facultad de Diseño y Comunicación, Universidad de Palermo. infocedyc@palermo.edu

Alicia Banchero: Lic. en Sociología (UBA). Profesora de la Facultad de Diseño y Comunicación de la UP. Integrante del Equipo de Directores de Proyectos 2004 del Centro de Estudios en Diseño y Comunicación, Facultad de Diseño y Comunicación, Universidad de Palermo.
} 
El presente trabajo pretende ser una indagación sobre las fronteras, límites de la creatividad, lo cual implica analizar los procesos creativos dentro de las prácticas y los discursos históricos en los diferentes momentos históricos. De esta forma pensaremos a la creatividad como un medio, como un atributo y como un instrumento con el que pueden ser caracterizadas y analizadas las actividades humanas, como lo son la ciencia y el arte, construyendo el propio camino del pensamiento de la creatividad. En definitiva será una reflexión sobre la creatividad, la creación y los creadores, como atributo, proceso y sujeto, enfocando ciertas disciplinas como el conocimiento científico, en determinados momentos históricos. Es decir indagar la relación entre el conocimiento y el arte, como proceso creador y las propias posibilidades históricas y sociales de la creación.

Por otra parte, será un intento por pensar a la creatividad desde un enfoque sociológico, encuadrándola dentro de la producción de sentido, sentido que tiene origen y destino en lo social. Pero ese sentido no existe de manera independiente. Tiene siempre un punto de referencia en el bagaje de experiencias previas. Lo social tiene una doble existencia: se expresa tanto en lo subjetivo como en lo objetivo. El poder simbólico se juega entre lo objetivo y lo subjetivo entre estructuras y esquemas de percepción de pensamiento y de acción. Es decir que toda producción para ser analizada, se encuadra dentro de la producción de sentido de la época. Lo que implica analizarla desde su historicidad.

Un discurso es un deambular de proposiciones dotadas de sentido que los individuos comparten de acuerdo a sus roles y a su posición en las instituciones. Para que un discurso sea eficaz debe conseguir ciertos objetivos, los suyos, y para poder arribar a ellos tiene que existir un aval en las prácticas. El campo es lo social hecho cosa, es una estructura objetiva, pero lo social también está inscripto en los cuerpos y determina formas de actuar. Podemos decir que los campos en los que se dan las relaciones en el ámbito del conocimiento científico o del arte, implican determinadas prácticas concretas a partir de ciertos esquemas de percepciones y categorizaciones que se aprehenden de la realidad. Teniendo en cuenta la conceptualización y el análisis propuesto por Pierre Bourdieu en cuanto a las estructuras objetivas que estructuran el accionar de los grupos y de los individuos, no en todas las épocas son dichas y practicadas las mismas prácticas y los mismos discursos. Esto es, las exclusiones se apoyan en lo que el dispositivo de discursos y prácticas permite. En términos de Bourdieu lo que el campo permite.

El creador crea desde /sobre el límite que imponen las relaciones sociales, desde el sentido que ellas imponen. Son quizás creadores, pregunta que guía la indagación, los que se topan con ese límite, esa frontera, impuesta, interna y externa, ese sentido; los que se atreven a ver el mundo desde otro lugar, desde fuera del pensamiento normal de la época, sin tener en cuenta el valor de uso del producto creado, sin que esa misma creación sea producto.
Para que haya creación, debe haber ruptura, ruptura con lo previo, con la manera de ver el mundo, con el sentido, como construcción social. No es el mundo el que cambia sino la forma de mirarlo y esto depende del bagaje de experiencia previa. La nueva obra, el nuevo resultado, no puede concebirse aislado.

En la actualidad la sobrecomunicación, la problematización de la vida cotidiana, han variado el sentido de lo social, como bagaje previo para "leer" el mundo. El conocimiento científico ya no es el referente más importante de la sociedad. Este lugar de mundo nuevo, de incertidumbre, donde ya no hay supuestos dados, es también enmarcado por la evanescencia, por lo efímero, por la situación. Los mensajes son instantáneos. Entonces, cuál será el límite, la frontera que pretende cruzar la creación y el creativo en este contexto. En definitiva qué condicionantes del orden de lo simbólico, cultural, posibilitan o bloquean la creatividad.

Para ilustrar, baste con un primer ejemplo que relacione las posibilidades históricas para que un conocimiento pueda ser desarrollado creativamente: el hallazgo de hipótesis que implicaban que la tierra girara, había existido previamente a su "imposición", su transformación en Ley, pero no había podido encuadrarse dentro de las prácticas y valores de la época. En la época en la que el peso de Dios como centro cambia, los enunciados también lo hacen. En la época medieval las prácticas y los discursos respondían a una estructura social tripartita, estructura que no permitía la proliferación de conocimientos o de prácticas que no estuvieran avalados por la idea central de un Dios unívoco. En ese contexto se producían discursos y prácticas, valores y apreciaciones que sólo eran aceptadas dentro de ese sistema de valores. Si bien el pensamiento creativo o lo creativo del pensamiento existían, y en este caso tomaremos la noción de creatividad ligada o una mirada diferente, muchas veces morían en la hoguera. En palabras de Thomas Kuhn, los paradigmas, esto es el modelo de creencias, valores, técnicas que comparte una comunidad científica, o podríamos decir en términos de Bourdieu, el campo, no permitía y expulsaba, de una manera drástica por cierto, al pensamiento distinto. Cabe aclarar también que esos valores compartidos generalmente, y más aún en lo cotidiano, se dan por supuestos sin ponerlos a prueba y casi sin conocerlos, sólo transcurriéndolos. Las prácticas, entonces, se dan dentro de un contexto de sentido de verdad, que está construido por ese sistema de valores de la época. Allí, en ese espacio y tiempo se define, se acepta o no la práctica. Los que no se circunscriben a esos valores no están dentro sino fuera, o son expulsados.

Lo que en un determinado momento y espacio puede ver un hombre, puede conocer y puede crear como sujeto, depende de lo que mira pero también de lo que su experiencia visual y conceptual previa lo ha dispuesto para ver.

Aquí no se planteará la noción de la creatividad como un concepto ligado a la eficacia ya que hacerlo implica observarla desde una visión puramente utilitaria. Cuando la dimensión económica hegemoniza el 
análisis la visión utilitaria de la creatividad aparece ligada a la resolución de problemas, aparece siendo una herramienta que responde sólo al mercado, dentro de relaciones que como fin tienen un beneficio, que se valorizan en el intercambio.

La creatividad aparece entonces como una estrategia para llegar a una respuesta o para responder a un problema o hallarle la solución. Desde este punto de vista, es decir el que la liga a una visión puramente eficaz, de resolución de problemas, se plantean momentos de la creación, momentos que van desde la incubación hasta la verificación. La misma posibilidad de asignarle momentos a proceso creativo implica segmentarlo en partes en las que el análisis de datos y su procesamiento juegan un papel importante. Cabría preguntarse si los "creativos" 0 los "creadores" pensaron y piensan en la posibilidad de utilizar su creación o simplemente buscaron preguntas a ser respondidas, o se enfrentaron precisamente con aquello que luego se transformó en un "útil" o en otro objeto.

Karl Polanyi ya había advertido en los años cuarenta que el hecho de permitir al mercado dirigir únicamente al destino humano era un mecanismo que implicaría la destrucción de la sociedad. Y en este sentido podemos decir que la expresión alude a la sociedad como una esfera de redes y de símbolos, en los que los procesos son significados por los individuos, sin que esto sea conciente. En este sentido dejar todo actividad humana a la esfera unidimensional de la economía es por un lado un análisis miope de la realidad y por otro lado el peligro de que la utilidad sea pauta de toda medida, aún del arte.

Kuhn postula la idea que los científicos no buscan estrictamente la verdad en un proceso acumulativo de conocimientos sino más bien, la búsqueda se centra en una sucesión de realizaciones reconocidas que durante algún tiempo brindan soluciones a problemas. En todo caso la creatividad ligada a lo artístico, con lo que tiene de histórico, no permite un análisis sólo desde un enfoque utilitario, aunque ciertas teorías sobre el hecho artístico así lo proclaman. En su teoría estética Adorno, pensando en la obra como construcción ligada a lo social, plantea: "Sin embargo, la comunicación de las obras de arte con el exterior, con el mundo del que por suerte o por desgracia se han cerrado, se da por medio de la comunicación y en ella precisamente aparecen como refracciones del mismo. Es fácil pensar que lo común entre su reino autónomo y el mundo exterior son sólo elementos que ha pedido prestados y que entran en un contexto completamente distinto. Sin embargo tampoco se puede discutir esa trivialidad perteneciente a la historia de la cultura de que el desarrollo de las conductas artísticas tal como se resumen por lo general bajo el concepto de estilo, se corresponden con el desarrollo social. La obra tiene una historicidad. Las fuerzas de producción estética es la misma que la del trabajo útil y tiene en sí la misma teleología y lo que podemos llamar relaciones estéticas de producción todo aquello en lo que se hallan encuadradas las fuerzas productivas y sobre lo que trabajan, no son sino sedimentos o huellas de los niveles sociales de las fuerzas de producción" (Adorno, T.1970: 15) El análisis que aquí se intentará estará más ligado al contexto en el que se producen innovaciones y al porqué de ellas, dentro de las prácticas y los discursos de las épocas. El recorrido que se intentará comenzará por una indagación de la ciencia y su desarrollo como proceso histórico, y por otra parte el papel que ha jugado y juega el método en ese camino. Luego la indagación se instalará en la posmodernidad y su paradigma.

\section{Ciencia y creación: El lugar de las prácticas científicas. El pensamiento de Thomas Kuhn y Paul Feyerabend}

La discusión que se instaló sobre la historia de la ciencia, en la que Paul Feyerabend y Thomas Kuhn fueron algunos de sus protagonistas, nos servirá como soporte para indagar las cuestiones ligadas al método y lo "permitido" en cada época, es decir, nos permitirá pensar en el método y los paradigmas, entre otras cuestiones, como motores y lugares de la creación desde el enfoque la ciencia.

Thomas Kuhn propuso que la historia de la ciencia debe concebirse a partir de las revoluciones internas, interpretadas como rupturas con la ciencia anterior. Cada vez que en la historia de la ciencia hay un quiebre con lo anterior, con la ciencia normal, se devela " otro mundo" . El "descubrimiento" del viaje de Colón, le indicó que sólo había llegado a las Indias.

Precisamente era eso lo que esperaba descubrir. Entonces, eso fue lo que pudo ver. Ese nuevo mundo, que tiempo después fue conceptualizado así, no sólo hacía referencia a un nuevo continente, nuevo desde el punto de vista de lo desconocido, sino que también hacía referencia a un nuevo modo de mirar, de observar, de conocer. Entonces, el descubrimiento, no es algo inmediato. "Para descubrir algo, para captar un fenómeno nuevo, las categorías conceptuales deben estar preparadas de antemano, de lo contrario se lo asimilará a lo ya conocido o se lo desconocerá" (Plax, J, . 1996: 48). Entonces cabría preguntarse por el lugar de la creatividad y de la creación como el proceso de un sujeto, el lugar de la libertad, por un lado, como planteará Feyerabend, y el lugar impuesto, externo al individuo. Por otra parte pienso: ¿Que hubiera pasado si Colón hubiera querido realizar su viaje para descubrir un nuevo mundo? Las condiciones estaban dadas para asimilar la idea de la redondez de la tierra, sólo eso. Lo conocido, encuadrado en el sistema de prácticas y discursos, era lo posible. Otro ejemplo que propone, entre tantos, es el descubrimiento de los rayos $X$, y en este sentido cabe aclarar que cuando habla de descubrimiento se refiere a un proceso de asimilación conceptual que, en algún sentido involucra también al cambio de paradigma. Como decíamos, plantea que el descubrimiento de los rayos $X$, corresponde a un hallazgo a través de un accidente. Pero debido precisamente a esta contingencia cabe preguntarse cuando realmente se produce el descubrimiento y por otra parte, particularmente este descubrimiento no perturbó al paradigma de la época, como lo hizo por ejemplo el 
descubrimiento del oxígeno. El encuentro con los rayos $X$ por parte de Roentgen se dio en momentos en los que la tabla periódica de elementos estaba incompleta. De todas maneras creó ciertos conflictos en la comunidad científica. En definitiva los rayos contribuyeron finalmente a la ciencia normal, abriendo un nuevo campo, y a su vez, modificando campos que ya existían. Pero no todas las teorías pertenecen a paradigmas. Muchas veces los científicos desarrollan ciertas teorías que denomina especulativas y que no están articuladas entre sí. De todas maneras, sólo cuando un experimento y la teoría "de tanteo" coinciden, surge un descubrimiento y allí, sólo allí la teoría se convierte en paradigma. El proceso es complejo e implica muchos factores.

Siguiendo a Kuhn, en ocasiones, cuando se descubren nuevos fenómenos pueden aparecer nuevos conceptos y categorías, y en definitiva un nuevo paradigma. Esto se da cuando la ciencia normal, que es la que tiene capacidad de explicar en un contexto, comienza a ser objetada. Entonces cuando todo parece no encuadrar es cuando esa ciencia normal y sus paradigmas no pueden explicar (no olvidemos que explicar es uno de los objetivos de la ciencia moderna) y estas disposiciones se vuelven cada vez más complicadas y de alguna manera muy forzadas y las hipótesis son cada vez más acotadas a los problemas que se pretenden resolver; todo este proceso, para que la teoría no se venga abajo.

Entonces la ciencia normal ya carece de valor explicativo, ya no encuadra las explicaciones y se necesitan otras maneras de explicar los fenómenos, otra forma de ver el mundo y de explicarlo. Se produce la crisis de la ciencia normal, y luego de la crisis, la nueva ciencia normal no será una transformación de la anterior, sino su sustitución

Kuhn ilustra el párrafo anterior: "Al mirar la luna, el convertido a la teoría de Copérnico no dice: antes veía un planeta, ahora veo un satélite. Esta frase implicaría un sentido en el que el sistema de Ptolomeo hubiera sido correcto alguna vez. En cambio alguien que se haya convertido a la nueva astronomía dice: Antes creía que la luna era un planeta (o la veía como tal); pero estaba equivocado".(Kuhn, T. 1962: 181). Lo impuesto de las prácticas y los discursos es tan relevante que sólo hay lugar a la equivocación para explicar un nuevo concepto. Lo anterior ya no sirve. Ahora es la verdad, ahora es lo correcto. Pero para que esto ocurriera, en un proceso al interior del ciencia, aparece la figura necesaria del que comienza a desarrollar el pensamiento que luego será el verdadero, aquel que comenzó a pensar y a permitir la suposición de que el sol era el centro de un sistema, tal y como lo aceptamos actualmente. El que se desvió y en un determinado momento fue expulsado, hasta que las condiciones, el campo, se consolidó y la forma de explicar el mundo nuevamente cambió. En este sentido el proceso del desarrollo científico es gradual, pero cuando la llamada ciencia normal no puede explicar más los fenómenos, es decir, cuando las disciplinas ya no pueden distraerse sobre los huecos explicativos de las teorías se produce un proceso que por fin concluye en un nuevo conjunto de prácticas y compromisos, una nueva base para los científicos. Este proceso es el que Kuhn concluye como Revoluciones Científicas, el motor del conocimiento científico.

Los parámetros y los principios de las ciencia normal especifican tanto los componentes del Universo como aquellos que no contiene, esto es que no sólo plantea las características y los lugares de los objetos sino que también aquellos objetos y su ubicación que va a considerar como verdaderos y aquellos que no lo son. Es por eso que caracteriza a los paradigmas como un conjunto de ilustraciones de diversas teorías en sus aplicaciones conceptuales, instrumentales y de observación. Es por eso que puede ocurrir que el interesado por la historia de la ciencia y su desarrollo se vea tentado a pensar que cuando cambian esos modelos a los que Kuhn llama paradigmas, es el propio mundo el que cambia también y conjuntamente con el; sin embargo ahora, con nuevos instrumentos (pensemos en el telescopio Galileo) intentan buscar en lugares en los que hasta ahora (con la ciencia normal aún sobreviviente) no se había buscado, y esos lugares son nuevos. Pero también son nuevos, en el sentido de nueva visión, los lugares en los que otros ya habían mirado, pero vieron otras cosas. Esto ocurre porque los científicos "responden" a un mundo diferente En este sentido podemos decir, parafraseando al mismo Kuhn, que lo que puede ver un hombre, en este caso un científico, depende de lo que mira, pero también de lo que la experiencia le permite ver.

Analizar a la ciencia como proceso en el que se juega la creatividad implica también relacionarla con lo exterior a ella, con lo que tiene de sociológico ese análisis, sus límites, y los condicionantes sociales y económicos, y los procesos subjetivos que hacen que se desarrolle este tipo de conocimiento, lo cual implica acceder a las consecuencias y a los propios actos del desarrollo científico.

De alguna manera investigar es buscar, ir más allá de lo que se ve, mirar y observar creativamente. Es aquello que se opone al sentido común. Se opone en cuanto a la falta de método en la búsqueda del conocimiento y en cuanto al origen de la información que explica. Y esto nos lleva a pensar en el papel del método en esa búsqueda. ¿Qué es primero? ¿La búsqueda o el método?. Cuando miramos, lo que vemos varía según lo que sabemos, pensamos o esperamos. Por ello el simple mirar implica un previo adiestramiento, experiencia o conocimiento. Sin embargo, muy a menudo no nos damos cuenta de ello, y así atribuimos nuestras observaciones enteramente a la cosa objeto de observación y no a cierta combinación del objeto y el observador (nosotros mismos). Y dado que no nos damos cuenta de nuestras inclinaciones, no las tenemos en cuenta. "El simplemente mirar supone presuntuosa ignorancia". En este contexto se puede decir que toda observación presupone una teoría previa, es decir, ciertas categorías en las que encuadrar nuestro nuevo conocimiento.

Kuhn planteará desde la epistemología, los aspectos más sociológicos e históricos de este tipo de conocimiento. Algunos han llamado a este tipo de postura las "nuevas epistemologías" En este sentido, esta 
orientación analiza a la ciencia desde un punto de vista sociológico, dejando en evidencia la relación de la ciencia con el contexto, como así también hacia el interior de su desarrollo. El propio concepto de paradigma remite a un análisis de este tipo ya que por un lado vincula a la aceptación de ese nuevo paradigma hacia afuera y hacia adentro. Además, el paradigma impulsa al nuevo conocimiento científico. Como ya se dijo, no hay en ese sentido una búsqueda especifica de la verdad, sino una búsqueda estratégica de solucionar problemas. Además, el paradigma da forma a la manera de ver el mundo. El paradigma, entonces, permite moverse, permite ver, encuadrar sin ponerse en duda su grado de verdad, ya que es en si mismo verdadero.

Las etapas en el desarrollo de la ciencia para Kuhn son:

- El momento Pre científico. Lo característico de esta etapa es que la actividad científica está fragmentada en muchas teorías e investigadores diversos y no hay unanimidad de los instrumentos que se utilizan para investigar. Es decir que no hay una fuerte comunicación entre la comunidad científica y las discusiones son más filosóficas que científicas. Para encontrar un ejemplo, puede ser esta la situación de las ciencias sociales en muchos casos. En definitiva no hay un dominio de ninguna teoría.

- El momento de logro. En esta etapa surge algún científico que formula una teoría, o descubre o inventa algún instrumento que aparece como exitoso a la hora de investigar.

- El momento de conversión. Aquí hay un convencimiento de la comunidad científica por la solución propuesta en la etapa anterior. Kuhn sugiere que este convencimiento no se funda en argumentos racionales.

- El inicio de la ciencia normal. Es aquí donde se introduce el concepto de paradigma. Este alude al logro científico que es aceptado por la comunidad y en general plantea dos aspectos, uno ligado a sus condicionamientos sociales y económicos, en el sentido de ser o no aceptado por la comunidad científica y por el otro lado responde a un aspecto valorativo en cuanto a la posibilidad de resolución de problemas. De todas formas podemos pensar al paradigma como una especie de motor impulsor de investigaciones y de indagaciones posteriores. De todas maneras Kuhn piensa a la ciencia como una actividad que sirve para la resolución de problemas más que como una actividad ligada al pensamiento verdadero, en tanto búsqueda. El que proponga una mejor solución, colaborará al desarrollo científico, colaborará mejor al desarrollo de la revolución científica. Igualmente cabe aclarar que el paradigma no es un aspecto que sea reconocido, sino que más bien tiene un fin utilitario, pero que no es notado. Por otra parte tampoco se discute, ya que solamente aparece explícito cuando se produce la crisis de la ciencia normal y ya deja de poseer la misma estructura.

- La fase de las primeras anormalidades, crisis y revolución científica. Las anormalidades surgen en el sentido de no poder articular ciertos aspectos de la investigación con el paradigma. En realidad, la primera reacción de la comunidad científica es rechazar esa irregularidad; pensamos en el recurrente ejemplo de Galileo Galilei. En un principio esas anormalidades no son tenidas en cuenta como fallas de la teoría, sino más bien como fallas del científico, ya que no son reconocidas por la comunidad científica, todo el peso del "error" cae sobre el científico y no sobre la teoría. No hay un científico creativo, hay un científico equivocado.

- El comienzo de la crisis. Aquí las irregularidades son más frecuentes y ya comienza a ser difícil soslayarlas; esta situación genera cierta insatisfacción en la comunidad científica aunque aún no se termina de abandonar el paradigma de la ciencia normal.

- El advenimiento de la emergencia. En esta etapa el paradigma comienza a peligrar en su persistencia y comienza a ser mirado críticamente por la comunidad. Comienzan a aparecer los subparadigmas, en un resquebrajamiento del paradigma. Aquí comienzan a aparecer discusiones epistemológicas sobre ciertos fundamentos de las teorías.

- Nueva fase de logro. Esta es una etapa similar a la descripta como etapa de logro. Si bien no se abandona definitivamente el paradigma por una cuestión de "practicidad".

- Nueva fase de conversión. Aquí comienzan nuevamente las etapas, tal cual fueron descriptas anteriormente, hasta llegar a:

- La nueva ciencia normal

La decisión de rechazar un paradigma surge entonces, de la comparación de el paradigma anterior con el nuevo paradigma y la comparación de ambos con la naturaleza, en la contrastación.

Entonces, el avance de la ciencia es discontinuo. Para Kuhn es importante el bagaje de creencias y valores a la hora de caracterizar el proceso científico. Se le ha criticado que es irracional en su análisis ya que no acepta la razón de un proceso lógico y neutral en la ciencia que permite comparar las teorías para decidir cual es la mejor. En cuanto al papel de la verdad, queda relegado, ya que cada paradigma, como dijimos, construye su propia verdad y realidad, con lo cual no habría una realidad objetiva. Los nuevos paradigmas son mejores, no sólo porque son verdaderos, sino porque pueden dar respuestas a los requerimientos de la época, en un contexto de sentido. Encontramos entonces una relación entre el motor de la ciencia con unos objetivos, que si bien caóticos, discontinuos, por fin se establecen y perduran, hasta una nueva crisis.

Kuhn, posteriormente va a plantear algunas modificaciones a su concepciones, luego de algunas críticas recibidas, sobretodo relacionadas a la ambigüedad del concepto de paradigma. De esta forma va a terminar concluyendo que sólo debe tomarse el concepto como:

- matriz disciplinar, esto es como sistema de creencias y aptitudes instrumentales compartidas por una comunidad científica y como:

- ejemplar, esto es un ejemplo que sirva de guía, por imitación o inspiración, para la actividad de investigar. 
Reconoce también que ver el paradigma como un "estilo" de investigación es algo poco claro. Tampoco es sencillo determinar qué es una "comunidad científica" y cuáles son sus límites. Con ello, Kuhn se ve obligado a pensar que pueden haber muchos paradigma "pequeños", en oposición a su idea previa de 1962 sobre los grandes paradigmas.

Ahora bien, el método científico, sin entrar a debatir su conformación o su lógica, puede pensarse como la " plantilla" con la que se hace ciencia. Sin embargo, una postura más anárquica implicaría pensar que, como el método a lo largo de la evolución de la ciencia ha fracasado, fracasará siempre, por lo que no es ese el mejor encuadre del conocimiento., sino que puede ser utilizado cualquier forma y recurso en la ciencia. Esta es muy básicamente parte de la postura de Paul Feyerabend a la hora de postular su anarquismo metodológico. El método científico se transforma entonces, separado de la historia, objetivo, con una lógica propia. Su propósito será entonces la búsqueda de la uniformidad. La ciencia queda afuera del análisis histórico.. Los hechos científicos son explicados lejos de las opiniones, las creencias y de la cultura. Entonces, el papel del científico en la evolución de la ciencia es, para Feyerabend, la tarea de quienes no quisieron someterse a las reglas 0 , sin querer, no las cumplieron. Es decir, los que salieron del margen marcado por lo posible, lo posible dentro del contexto discursivo y de prácticas de la época. Acá se puede plantear una diferencia con Kuhn, mientras que este plantea que la ciencia normal, me atrevería a decir la ciencia permitida, deviene en una crisis cuando entran en contradicción sus paradigmas, Feyerabend plantea la multiplicidad de aspectos que deben tenerse en cuenta a la hora de hablar de nuevos descubrimientos o inventos.

La historia de la ciencia y del conocimiento científico no es lineal ni surge para la creación de problemas. Para Kuhn el paradigma no sólo es un conjunto de teorías, sino que es una forma de ver el mundo, una especie de ideología. Es por eso que la fortaleza de ese modelo, durante el transcurso de la ciencia normal, lo hacen difícil de derribar. De alguna manera es una estructura que se adopta por consenso y permite ver a través de él al mundo. También tiene la utilidad de ser el basamento de la resolución de problemas. Como ya se dijo, el paradigma es aceptado más por su utilidad que por su grado de verdad. La propia experiencia no rechaza al paradigma, de alguna forma obliga a articularlo nuevamente para que soporte las nuevas contradicciones. Pero en ese proceso el procedimiento termina haciéndose más fuerte ante los embates de las anormalidades.

Por otra parte Feyerabend, más allá de criticar la separación entre la historia de la ciencia, la filosofía y la ciencia misma, también se plantea la importancia de una perspectiva humanista, esto es la necesidad de dar importancia en el proceso del conocimiento a las creencias, los talentos particulares y a la imaginación, llevando el análisis a una comparación con el arte. "Es posible conservar lo que podríamos llamar la libertad de creación artística y aprovecharse al máximo de ella, no sólo como una válvula de escape sino como medio necesario para descubrir, y tal vez para cambiar los rasgos del mundo en que vivimos" (Feyerabend, P. 1975: 37)

La idea de pensar en un método como molde estanco para pensar, con reglas fijas y universales, no deja lugar para la creación a través del talento en las individualidades particulares. Por otra parte, no se toma en cuenta lo que hay de histórico en el desarrollo del conocimiento científico. El hecho de utilizar el método implica la aceptación de su perfección. La única regla que vale es la del "todo vale". También en este autor hay una marcada mirada sobre la relación entre ciencia y dominación, esto es la imposición de la lógica y de sus hallazgos, no en un contexto de libertad y armonía, sino de dominación por la fuerza. Esto también nos lleva a dos conceptos, como lo son el contexto de descubrimiento y contexto de justificación, que según Feyerabend son dos procesos que van juntos; esto es, de la manera en que el científico descubre, investiga y justifica, en ese contexto histórico, socio político, se dan ya las constrastaciones. Son dos procesos, que chocan, y que son analizados por la historia y la filosofía de la historia, separadamente. "Al inventar teorías y contemplarlas de un modo relajado y artístico damos a menudo pasos que están prohibidos por las reglas metodológicas" (Feyerabend, P. 1975: 154). Por otra parte no hay una distinción, un límite entre las teoría y las observaciones, en tanto que el aprendizaje no es un camino desde la teoría a la observación, sino que en el implica a ambos elementos. Lo que hay de creativo en el proceso de la investigación y el descubrimiento tiene que ver con la conexión entre el individuo y la ciencia. "En todos los casos la imagen percibida depende de los sistemas mentales que pueden cambiarse a voluntad, sin la ayuda de drogas, hipnosis y reacondicionamiento. Pero los sistemas mentales pueden quedar paralizados por enfermedad, como resultado de la inmersión de alguien en una cultura determinada, o por causa de determinantes fisiológicas, que no están bajo nuestro control... nuestra actitud hacia las otras razas o hacia personas de diferente trasfondo cultural, depende a menudo de sistemas paralizados de la segunda clase: habiendo aprendido a leer rostros de una manera standard, hacemos juicios standard y nos extraviamos" (Feyerabend, P. 1975: 218).

Comparando a los dos autores que venimos indagando podemos decir que ambos le dan a relación entre teoría y observación papeles similares. Lo que no se encuadra en lo que sabemos previa-mente pareciera que no existe, por lo menos de manera general. En todo caso a mayor libertad mayor posibilidad de comprender y de encontrar otras res-puesta y otros caminos. Si el encuadre es muy fuerte, no hay avance del conocimiento. Como ya se dijo para Feyerabend, el lugar de la creación científica es del todo vale, es el lugar de las creencias y los valores y es el lugar de la desviación, del no método, de la anarquía. La "irracionalidad" ha alimentado buena parte de los inventos y descubrimientos, buena parte de la creación en el conocimiento.

Ambos coinciden en el enfoque sociológico que debe 
tener una historia de la ciencia, sin ser separada de la filosofía de la ciencia. Feyerabend plantea la necesidad de crear, de no responder a la dominación. Para el método ha fracasado. Cabe la pregunta: ¿Es posible crear con un método? ¿O el método es la antítesis de la creación?. Creemos en este sentido en que lo dogmático, aún en lo científico, es contraproducente a la creación. Por otra parte los descubrimientos científicos muchas veces fueron creados sin método, casi fortuitamente (recordemos el caso de los rayos $\mathrm{X}$ ).

Para este autor el científico que se interesa por lo empírico y por las teorías debe abrazar una metodología pluralista, y esto implicará ser amplio desde el punto de vista de la comparación entre teorías. Y en este sentido, nuevamente, to do vale: el acercamiento al conocimiento puede hacerse a partir del pasado, como por ejemplo en los mitos antiguos. Así como propugna la no separación entre la filosofía de la ciencia y la historia de la ciencia, también asume la no separación entre ciencia y no ciencia. Aún la locura puede ser un punto de partida de la "no metodología" . Como ejemplo ilustrativo de esta idea plantea que la idea del movimiento de la tierra, luego de Aristóteles y Ptolomeo, fue desechada completamente para ser retomada por Copérnico. Esto ocurrió porque las teorías fueron abandonadas antes de poder siquiera mostrarse, y más aún de mostrar su utilidad. Allí también encuadran ciertos mitos, que no son tenidos como verdaderos, ya que se desconocen sus contenidos científicos. Lo que no puede compatibilizarse con la ciencia, no vive, dirá Feyeraband. Es interesante entonces plantearse que dentro de este contexto en el que ciertas teorías no sobreviven al método científico, mañana puedan transformarse en teorías relevantes para la ciencia, ya que entran dentro de lo científico del momento. La creatividad en esta concepción se relaciona con una perspectiva humanista, como dijimos antes. La contradicción es la siguientes. Si bien se espera desarrollar las individualidades al máximo, particularmente en la enseñanza, para desarrollar talentos y creatividades, no se da lugar a la creatividad al "soñar despiertos" como fuente. "Es posible conservar lo que podríamos llamar la libertad de la creación artística y aprovecharse al máximo de ella, no sólo como una válvula de escape sino como un medio necesario para descubrir y tal vez, para cambiar los rasgos del mundo en que vivimos" (Feyerabend, P. 1975:37)

Hacer coincidir o buscar la relación entre lo individual y "el mundo en que vivimos", esto es lo subjetivo y lo objetivo, es una de los soportes argumentativos de la metodología pluralista, como llama el filósofo a este enfoque.

Por otra parte el contexto de descubrimiento y el contexto de justificación son para Feyerabend dos aspectos del proceso histórico de la ciencia, y es precisamente instalar una discusión desde una perspectiva histórica. De no ser así, los enunciados se podrían comparar sin tener en cuenta su historicidad $y$, entonces a partir de una teoría, como conjunto de proposiciones relacionadas, se pueden extraer diferentes hipótesis, dando por supuesto que las observaciones y las teorías deben ser utilizadas o para afirmar o para rechazar la hipótesis, sin tener en cuenta el contenido histórico de esa teoría y a la vez de las observaciones. Las teorías están conformadas por elementos expresados explícita y claramente pero otros son ocultos y sólo son sacados a la luz posteriormente. Para aceptar o descartar una nueva hipótesis se deberá tener en cuenta la situación histórica. Citaremos como ejemplo la concepción aristotélica del conocimiento y la percepción, y la hipótesis geocéntrica: ambas se adaptaban perfectamente ya que la percepción para Aristóteles, es un proceso en el que la forma del objeto observado que se percibe pasa al perceptor exactamente según la misma forma que caracterizaba al objeto, de manera que el perceptor, de alguna forma, asume las propiedades del objeto. La experiencia es importante para el conocimiento en esta concepción, ya que la percepción del que observa contiene las mismas formas que tiene el objeto. Por lo tanto desde esa perspectiva no existe posibilidad de alguna distancia entre las observaciones y las cosas observadas. Para modificar esta observación será necesaria una nueva concepción del mundo, de sus hombres y de las formas de conocer. Sabemos que la ciencia como la conocemos, con el advenimiento de la modernidad, comienza con Galileo Galilei. Que es precisamente quien quiebra la explicación tradicional aristotélica. Para Aristóteles el hombre no tiene incidencia en el movimiento del universo. La tierra ocupa un lugar central y los demás cuerpos que conforman el universo giran alrededor de ella. En esta concepción todos los cuerpos tienden a la perfección, fin último de los seres. Posteriormente a Aristóteles, Ptolomeo, aporta cambios a esta teoría con nuevas observaciones de los cuerpos. Sin embargo en el siglo IV a.C., Heráclides y después Aristarco de Samos afirman ciertas conclusiones relacionadas al heliocentrismo. Sin embargo durante muchos siglos no se consideraron pensamientos verdaderos. Los aportes de Copérnico son realizados tanto desde las observaciones como desde su concepción platónica. Luego de los aportes de Kepler, irrumpe Galileo Galilei, no sin resultarle compleja la tarea de aseverar, telescopio por medio, la teoría heliocéntrica. La iglesia, internada en esa época en la Reforma Protestante, se atribuía la autoridad capaz de interpretar las sagradas escrituras. La supervivencia de Galileo tuvo relación directa con el abandono de sus ideas, bajo la amenaza de la herejía. Baste este ejemplo para ilustrar mínimamente el contenido histórico de la teorías y las observaciones.

Para Feyerabend lo realmente creativo es lo artístico, sobre todo habiendo comprobado que las observaciones contrastadas con los enunciados no siempre resultan y es allí donde el enfoque apunta a otras alternativas relacionadas con el estilo, el atractivo, a partir de las cuales crear y aportar a una teoría que se desvió de lo empírico y que perdió carácter frente a teorías rivales. En definitiva, plantea una crítica contra el modelo hipotético deductivo y al Falsacionismo popperiano.

Si comparamos ambos pensamientos, el de Kuhn y 
el de Feyerabend, una de las distinciones que podemos encontrar es que el primero aduce que si bien los paradigmas son compartidos durante el transcurso de la ciencia normal, esto no implica compartir las reglas. Por otra, ambos le asignan una importancia relevante al conocimiento adquirido en la práctica. El encuadre de la ciencia normal en el caso de Kuhn, como del método en el caso de Feyerabend, en distintos grados por cierto, no permiten la realización total de la creatividad, en el sentido de fuerza que impulsa al descubrimiento. Por una parte si bien el motor de la ciencia son los paradigmas, el proceso de su conformación aparece como inhibidor de ciertas fuerzas creadoras. Sólo luego de todo el proceso que culmina con una nueva ciencia normal, es cuando se retoman algunos enunciados que antes no habían podido ser siquiera expuestos a la comunidad científica. De todas maneras hay una idea de avance, que si bien no busca la verdad, si funciona como una forma de ver el mundo, casi como una ideología científica. Por otra parte, el papel asignado al "no método" plantea una postura en la que los disparadores de los descubrimientos o los hallazgos son muy, quizás demasiado, amplios. La idea que subyace entonces es la de la plena libertad y de movimientos que permitan un avance, aún me atrevería a decir mayor, del conocimiento científico, ya que las trabas se acotan. De todas formas la internacionalización de lo objetivo, en subjetividades particulares reducen la posibilidad de la libertad plena. La idea de que la ciencia tiene que regirse por "reglas fijas y universales" es irreal y perniciosa. Lo es porque, en el primer caso implica la visión demasiado pequeña del talento de los hombres y todo lo que rodea a esa circunstancia; por otra parte es a la vez perniciosa porque forzar reglas implica incrementar lo profesional, por sobre lo humano. Esta es la idea de pensar en los científicos, de cualquier área, como seres humanos, que en general no siguen reglas fijas para la vida. Por otra parte tampoco esto es saludable para la ciencia misma, ya que no tiene en cuenta que su desarrollo es un proceso de por si complejo, en el que las circunstancias históricas son influyentes, por cierto. Feyerabend plantea que cualquier regla metodológica se asocia a una suposición global, por lo que al usar esa regla se da por válida la suposición. "El empirismo da por supuesto que la experiencia sensible refleja mejor el mundo que el pensamiento puro. El elogio de la argumentación da por supuesto que los artificios de la razón dan mejores resultados que el juego incontrolado de nuestras emociones. Semejantes suposiciones pueden se perfectamente plausibles e incluso verdaderas. Sin embargo, ocasionalmente, deberían ser sometidas a contrastación" (Feyerabend, P. 1975: 290). Esto implica que habría que dejar el método para "ver que pasa". Esto sólo implica no acotarse, no limitarse a las reglas. La historia de la ciencia indica que muchas veces se utilizaron otras reglas, sin que por ello se rompiera la validez de las mismas. Evidentemente el papel asignado a la libre indagación es muy importante en el filósofo, ya que una investigación no debe ser guiada sólo por los estrechos "canales de las cosas ya sabidas" Por otra parte construir nuevas concepciones del mundo y un lenguaje ad hoc implica incorporar las teorías, los argumentos a un lenguaje futuro, argumentando con términos que aún no han sido explicados, y a sentenciar, argumentar sin reglas claras. Cabria plantearse si de alguna, forma el hecho de el no método no es un método en si mismo. En definitiva en una sociedad que se caracteriza por la libertad, los que piensan, los intelectuales, forman parte de una tradición más. En todo caso el papel de la sociedad en un contexto de libertad es encontrarle un sentido a cada una de las subjetividades. Esto no significa que la racionalidad, ligada al método sea la única manera de ver el mundo, sino que es una de las formas por donde mirarlo. Pero no es lo que ocurre en la realidad. La propuesta estaría ligada a una mayor democratización del pensamiento, en el se puedan tener en cuenta a las demás tradiciones, de la misma forma que cuando se aceptan mitos 0 razas, se lo hace a partir de una con la que se busca la igualdad, el parámetro.

En definitiva, el proceso del conocimiento científico es eminentemente creativo, ya que a través del método o suponiendo su falta, el enunciado de hipótesis y las observaciones, se componen de aspectos que hacen a lo innovador y a los hallazgos. Sin embargo se suele ligar el conocimiento científico a la objetividad, en tanto una de las características intrínsecas del mismo. Pero creemos que el papel de la mirada diferente, de la individualidad para observar liga íntimamente el trabajo del científico con lo creativo. El desarrollo de las ciencias, en sus diferentes áreas, y en mayor o menor medida, así lo indican.

\section{La posmodernidad: el lugar y el tiempo de la creatividad}

Hasta aquí se reflexionó sobre las posibilidades históricas para el descubrimiento, la creatividad en un enfoque sobre el conocimiento científico. Esto es las posibilidades, en tanto contexto y en tanto método, como dos aspectos básicos de la ciencia tal y como la conocemos en la modernidad. Pero otra mirada merece ser hecha atendiendo la complejidad en el campo del conocimiento y las comunicaciones en la actualidad. Para ello pensamos en el quiebre que se produce con el desarrollo de la tecnologías, proceso que comienza a mediados del siglo pasado para transformase en la actualidad en un proceso aún más vertiginoso.

En el momento de la modernidad, como momento histórico en el que emergen ciertas prácticas y ciertas formas de conocer, aparece la noción de sujeto de conocimiento separado del objeto, en donde éste tiene la función pasiva de dejarse conocer. Como imagen podríamos decir que el conocimiento empuja hacia adelante, de alguna manera hacia la utopía. Una utopía que también está teñida por la posibilidad de conocer para dominar, para dominar a ese objeto de conocimiento. Esto también está totalmente relacionado con la idea de un futuro, y de un futuro "racional", basado en el desarrollo científico. Sin embargo, "el desarrollo de la razón, lejos de conver- 
te en motor de la liberación, se convertía, cada vez más en factor de dominación. Tal como lo señalaron la Escuela de Frankfurt primero y Jürgen Habermas después, la razón política, asimilada a la razón científica, devino ideología. Terrible paradoja para un proyecto que, como moderno, intentó hacer de la crítica su mayor bastión" (Díaz, E. 1996: 23). .

La emergencia de la posmodernidad, como proceso complejo que surge en el siglo XX y que continúa, nos muestra una faceta que trataremos de analizar. La principal característica de la actualidad es la de la diversificación de los conocimientos. Por ende la búsqueda es permanente. Lo supuesto, lo dado, ha perdido su valor. El mundo, la sociedad, la vida y la identidad personal son cada vez más problematizadas. Cada área es objeto de múltiples interpretaciones y a su vez cada interpretación implica determinadas esferas de acción posible, lo que complejiza aun más el panorama. "La posmo-dernidad se mira en el espejo del presente. Un presente que refleja la convergencia del pasado y del futuro. Y se mira asimismo en un espacio en el que coinciden todos los espacios (reciclaje de edificios, coexistencia de distintas modas, collages y pastiches). Multitemporalidad. Multiespacialidad. Ambas características circulan por todo el entramado cultural, aun por aquellos espacios de desgarro y dolor. Todo convive en la multiplicidad, no sólo las costumbres, la moda y el arte: también la ciencia. Ya no hay un sólo paradigma que agrupe a todas las ciencias naturales. Nunca hubo ninguno que agrupara a las sociales. El imaginario científico ha abandonado la pretensión de que una unidad subyacente estructura toda la realidad. Hoy se acepta la posibilidad de que la realidad responda al azar y a la indeterminación, más que al orden y a la objetividad. Lo que se perdió en supuesta seguridad se ganó en creatividad" (Díaz. E. 1996: 20). Creo que este párrafo describe con excelente justeza el acercamiento, y la confusión en algunos casos, en los que las prácticas ya no son delimitadas: ya no hay espacios o márgenes que caractericen a las prácticas, hay una convivencia, que por otra parte permite el acercamiento entre formas de conocimiento, o de creatividad que en otras épocas no se hubieran podido pensar. Por otra parte los cambios, en todas las áreas, son por lo mismo cada vez más vertiginosos y la función de los medios de comunicación, cada vez más extensivos Estos cumplen un papel fundamental en el imaginario, pensado desde el punto de vista de la legitimación que brindan (un científico para que diga una "verdad" es más creíble si la dice por TV... dentro del imaginario social).

La razón, la ciencia, como forma de conocimiento verdadero y demostrable, casi diría indiscutible, se diluye frente a lo fragmentado, lo vertiginoso, lo inmediato, que va perdiendo valor casi en el mismo momento. Pareciera que el espacio en el que se construye algo nuevo es más acotado, o de por si más efímero, en tanto espacio y tiempo.

En este sentido la posibilidad de comunicación ligada a los procesos informáticos plantea una forma de socialización y de posibilidades más intensas de acercamiento, en un principio, pero a la vez resultan más inabarcables.

Paul Virilio indaga sobre la situación actual en un universo técnico casi total y abarcador, que prácticamente es inexorable para el hombre. La pregunta que subyace a la obra de este autor sería algo así como examinar si le tecnología es un instrumento para alcanzar determinado tipo de bienestar o si realmente es un fin en si mismo.

El tiempo real de las redes de comunicación es el de la velocidad de una conexión mecánica e inmediata. La velocidad social no ha alcanzado su límite con las transmisiones informáticas y esto se relaciona con el poder, el poder del control, el poder político, el control del espacio y el tiempo. La velocidad y la proximidad no ayudan a la democracia, sino to do lo contrario. Si bien otros autores han mencionado que en la posmodernidad el gran relato ha perdido credibilidad, no es en la velocidad y en la inmediatez. El peligro para Virilio es la desurbanización, la desterritorialización en beneficio de la "coexistencia virtual de los ausentes". El real problema de la modernidad es basarse en el tiempo (el ahora) en detrimento del lugar (aquí). El lugar, ese "aquí" pareciera no existir más. " La reapropiación del cuerpo, para lo que la danza supone la resistencia máxima, no es simplemente un problema de coreografía sino un problema de sociografía, de relación con el otro, de relación con el mundo. De otro modo, es la locura, es decir, la pérdida del mundo y la pérdida del cuerpo» (Jiménez, R. 1998).

La problemática de la desterritorialización en la posmodernidad está íntimamente ligada al cuerpo, al lugar del cuerpo y al espacio del cuerpo como ámbito de la creación. El lugar del cuerpo aparece ligado a la falta de ocultamiento, como un sinónimo incluso de lo actual. Pero ese mostrase esconde el temor al propio cuerpo y al cuerpo del otro. También aparece ligado al lugar de la territorialidad de algunas demostraciones del arte y de la cultura. La obra artística puede ser considerada como una creación que está inscripta en un momento histórico. Es particularmente, a modo de ejemplo, en la obra literaria que al estar constituida por palabras, la inscriben en un determinado momento y en un ámbito geográfico.

En los años de las explosión de las expresiones de vanguardia, el futurismo y el cubismo, entre otras, se mostraban como expresiones de las utopías, esto es en la idea del descrédito del progreso indefinido. La ciencia como relato aglutinador de verdad comenzaba a desmoronarse. La incertidumbre, expresada también en el arte comenzaba a ser el signo de los tiempos. Las explicaciones comenzaban a debilitarse y la razón se topaba con procesos que no podía encuadrar. El paradigma de la modernidad ya era dudoso. Ese paradigma que en lento proceso sintió la influencia de la era industrial, y modificó la propia noción de belleza. Los cambios, lo nuevo comenzó a acelerar los tiempos, o al revés, y atrás fue quedando ese ideal armónico y trascendental. Como indicamos en cita de Adorno, en el arte se pueden ver reflejadas las problemáticas sociales de una época. Y en la actualidad, mediante el papel de los medios, entre otros papeles, se pierden aún más las fronteras y el 
arte, el show y el espectáculo son más difíciles de diferenciar. La pregunta sería si el arte debe sustraerse a algún código preestablecido. En Gran Bretaña Damián Hirst, un llamado artista contestatario ganó un premio con una obra que involucraba a una vaca y un ternero en formol. En Argentina, Cristina Piffer ha reflejado la violencia de la historia en mosaicos de resina transparente con carne.

Desde el punto de vista de las prácticas, del imaginario social, Esther Díaz plantea que surge la conveniencia de que el cuerpo del otro esté apartado, esté lejos, esté mediatizado. Lo real se teme pero a la vez se desea. Entonces eso que es tan temido se mediatiza, y se mediatiza de diversas maneras: el contacto ya no se realiza directamente con el cuerpo, la tecnología media entre los contactos, el teléfono, el chat, etc, y los medios de comunicación.

Paul Virilio entonces nos dirá que la deslocalización del hombre "posmoderno" tiene mucho que ver con la pérdida del propio cuerpo, consecuencia directa de la desaparición de la sociabilidad urbana física, tangible, real. Algo así como esconderse en el propio cuerpo, porque ya no queda lugar donde estar. Lo que realmente importa a Virilio de esta nueva coyuntura de la experiencia individual del espacio tiempo son las implicancias que tiene con respecto al modo de ver y crear. Lo que se pone en juego es una nueva visión del mundo, basada en una automatización de la percepción que amenaza directamente al entendimiento y al razonamiento. El nuevo paradigma de la época ya no permite la reflexión sino la inmediatez. Cabría preguntarse si esto es un buen ámbito para la creatividad, en cualquiera de las disciplinas que analicemos. Por lo pronto diremos que hay mayor cantidad de expresiones.

En cuanto a las expresiones artísticas particularmente, como expresiones creadoras del hombre Virilio afirma que el arte en definitiva ha sido derrotado o es cómplice de ciertas ideologías totalitarias del siglo $X X$. Por un lado la obra es efímera, es toda experiencia, es toda situación, las obras celebran lo instantáneo, la fugacidad. Este pensador plantea entonces una visión muy crítica, junto con otros como Polanyi, de este nuevo paradigma en el que la velocidad y la desterritorialización son básicos. Acaso esas características expresan el desprecio por el propio cuerpo. Las experiencias artísticas en el propio cuerpo en donde el cuerpo es protagonista abundan en este nuevo siglo y siguen planteando el interrogante a los pensadores sobre lo "transgresor" de las expresiones. Por lo pronto y siguiendo a Virilio, diremos que representan el desprecio por lo real. Lo real despreciado desde la esfera de lo social, no como un fenómeno individual.

Ahora bien, el cuerpo es también ámbito de relación, es decir hablamos de un cuerpo y del cuerpo del otro. En este sentido el cuerpo del otro aparece ligado al deseo y al temor, pero esta aparición tiene que ver con un aspecto de la época: el miedo de lo real, como expone Esther Díaz. Si bien en muchas épocas y momentos, los individuos rechazan la realidad, la característica de esta época es la "socialización del rechazo". Ahora ese rechazo tiene la posibilidad de ser global. La construcción de la realidad, desde el punto de vista de una construcción social, incluye prácticas y discursos que sustentan la "conveniencia" de la distancia con el cuerpo del otro. Acaso, "¿No es la estética del enemigo la que finalmente ha triunfado?", se plantea Virilio al analizar las obras de arte en las que el arte aparece ligado a lo orgánico, a lo corporal. "Es como si Narciso se hubiera animado a salirse de sí mismo pero, ante la angustia provocada por el cóctel del deseo y el temor, se retrotayera otra vez a si. " (Díaz, E. 1996: 326). En la posmodernidad, globalización mediante, la territorialidad pierde fuerza. El tiempo es otro. Se profundiza el vértigo. Como ya dijimos muchas expresiones artísticas tienen lugar en el cuerpo. Por otra parte la faceta contestataria o transgresora del arte aparece de alguna manera absorbida por el mercado. Lo que transgrede es negocio. Entonces cabría pensar que el arte vanguardista se institucionalizó. En este sentido aparece la provocación ligada al cuerpo, o a lo orgánico, al lugar en el que se puede operar, fuera de lo que está fuera, en el campo de la subjetividad. En los años noventa una actriz francesa filmó su propia operación estética, con intención de escultura, con el fin de inmortalizar la "obra maestra absoluta". Podría pensarse que el miedo al cuerpo del otro no es menor que el miedo al propio. Este episodio también muestra la relación entre arte, ciencia, tecnología y realidad, entre otros aspectos.

Desde un punto de vista sociológico el consumo de bienes culturales funciona como un diferenciador, esto es que el componente simbólico está presente a la hora de diferenciar y distinguir socialmente. Cabría preguntarse aquí por el rol del arte que en otra época pareció tener un efecto "educador" y comunicador de valores. Si el papel del arte varió, también habrá variado el significado de su consumo, esto es, la distinción que implica consumir estas nuevas formas de arte efímeras, encriptadas, tendrá otro significado y habrá perdido fuerza el anterior. Para Bourdieu el gusto, la preferencia y los estilos de vida tienen que ver directamente con la clase a la que se pertenece, por lo tanto insistimos en cuanto al consumo de esta nueva forma de arte y a las implicancias de la tecnología y su consumo ligada a la desterritorialización y al "rechazo de lo real".

Pero la pregunta sería ¿cómo salir de esta situación?, suponiendo que esta relación no aporta al avance desde lo creativo reflexivo, en principio en ninguna disciplina, y menos aun cuando aporta a la diferenciación social y aleja al arte de su faceta "educadora" . Por otra parte el rechazo del cuerpo, y en el uso de la tecnología como medio para el no contacto, llama desde la reflexión a pensar en ciertas modificaciones posibles. Virilio nos dirá por su parte que el valor de la palabra como lugar del encuentro y no del rechazo es una de las salidas. Por otra parte ya que la imagen está ligada al mundo de la afectividad, la palabra y el lenguaje permite una construcción de la subjetividad a partir del encuentro social.

La desaparición del razonamiento y el entendimiento contra la pura percepción, percepción para la que el tiempo absoluto es lo que el tiempo diferido, como 
distancia mediadora entre los hechos y la decisión, es a la razón. En realidad la idea es la recuperación de la ciudad física y real, incluso a través de la materialización del cuerpo y del mundo. Los referentes geográficos aparecen como importantes no como excluyentes ni segmentadores, en este pensador, sino más bien como referentes geográficos de las identidades. Identidad que recupera la historia del hombre.

La pregunta que nos guía ya casi al final es si existirá una correlación entre el arte y la territorialidad. Desde el punto de vista de la globalización esta es una forma de plantear, como ya hemos hecho, la desterritorialización de los procesos artísticos, como también de otras disciplinas. Cabría preguntarse también la ligazón existente entre cultura, como símbolos compartidos, y la relación con el arte. En tanto desterritorialización del arte, como de cualquier otra disciplina, pareciera ser que irá en detrimento de pérdida de identidad cultural. En todo caso la mayor homogeneidad no es conducente con una mayor creatividad en este caso. Si podemos decir que redundará en una considerable cantidad de expresiones. Por otra parte, como ya hemos dicho la virtualidad es la escena donde todo puede ser visto y compartido, sin posibilidad de demasiado juicio.

Por último nos plantearemos la pregunta sobre la relación entre territorialidad o espacio y la creación, es decir, si existe alguna relación entre la creación y los lugares, como espacios, de la creación, teniendo en cuenta el análisis anterior. En este sentido encontramos dos ejemplo paradigmáticos en donde se pueden encontrar y leer el sentido de las obras con los espacios en las que fueron creadas. Son los casos de Walter Benjamin y Immanuel Kant. El primero escribió su obra en un contexto de constante migración y permanente inquietud por causas políticas mayoritariamente, sin una residencia fija. Su obra marca esta característica peculiar de su vida, es fragmentada y en algunos casos contradictoria. En su producción reflexiona sobre la influencia de las grandes urbes, no solamente como lugares en los que la economía se mueve, sino como un lugar de cruces sociales, que, a la vez que posibilita la libertad del hombre lo aliena, en un ámbito de masificación anónima. La idea de ciudad en Benjamin está ligada a la urbanización acelerada, a los centros comerciales, por donde deambula el "flaneur". Así el flaneur nos remite a la exigencia necesaria para recorrer los espacios urbanos, marcando recorridos: los espectáculos, los parques temáticos, las exposiciones, los juegos, etc. Estos recorridos exigen a su vez un control de las emociones que sirva a la vez para sentir placer, algo así como un "controlado descontrol" de las emociones, que sirva para disfrutar del placer, la excitación pero a la vez para controlarse de las mismas. Vemos, a la vez, que su vida marcó de alguna manera las características de la producción, también las temáticas tuvieron que ver con recorridos, con la alienación y particularmente con la territorialidad.
Por el otro lado el otro filósofo alemán , Kant, nunca salió de su ciudad natal, por lo menos a grandes distancias, y su obra expresa coherencia racional que en parte indaga sobre el espacio y el tiempo como formas puras de la sensibilidad. Si bien esto puede no resultar una evidencia generalizable resulta interesante como marca que relaciona el espacio y la creación con algún enlace.

\section{Algunas reflexiones finales}

Se indagó por un lado ciertas características de la "creación" en la ciencia. Las posibilidades de esa creación ligadas a las probabilidades de lo permitido por la ciencia normal y a las distorsiones provocadas por ciertos enunciados que en definitiva desembocan en nuevos paradigmas. En esa concepción, el papel de la creación es amplio, ya que el "trabajo" de los científicos es la búsqueda permanente, traducida en un proceso que se repite. Por otra parte se indagó en el papel de freno del método para la creación y los descubrimientos científicos: el método, cualquiera sea este, inhibe la creatividad. El vale todo es el único medio para conocer, y hasta el arte puede ser disparador de la actividad científica. El papel de seres humanos es también aquí fundamental, ya que se toma a los científicos como tales y no puramente como profesionales, escindidos de su esfera creativa. Por otra parte la idea fue acercarnos a la actualidad, en un proceso que comenzó en el siglo $X X$, y que tiene que ver con planos en los que la confusión no permiten encuadrar límites ni fronteras, para las disciplinas y para las identidades. Este panorama deja muy atrás a la Razón como fuente de todo argumento, y la ciencia como su soporte. En definitiva se trató de ligar a la creatividad, en tanto un proceso individual y un proceso ligado a las prácticas y los discursos en tanto límites: el método, la ciencia normal y sus enunciados, el cuerpo, la tecnología. Los lugares en los que es posible la creatividad y cuando decimos lugares no sólo nos referimos a los espacios físicos, sino a los sociales, a esos lugares que antes eran expresados directamente por los espacios de la ciudad, sin virtualidad mediante.

La modernidad trajo la idea del progreso indefinido, del futuro, sin embargo esto no ocurrió así y la confusión borró fronteras. Hoy nada es seguro. En el propio cuerpo se puede crear, pero el límite del propio cuerpo es el cuerpo del otro, al que no me atrevo, al que rehuyo. La tecnología hace lo demás. Por otro lado la mercantilización de todo proceso creador ha hecho perder la idea de lo creativo ligado a subvertir órdenes y a romper con lo anterior. La novedad ahora pasa por otros lados.

Cuando decimos creativo deberíamos pensar si estamos pensando en el ser libre que proponía Feyerabend para conocer, o bien en el que es capaz de utilizar una herramienta de una manera útil. En el juego entre la inmediatez y lo que vendrá se juega el papel de la creatividad, tanto en el ámbito de lo científico como en el del arte, en definitiva de lo nuevo. 


\section{Bibliografía}

Adorno, T. W. (1983) Teoría Estética. Madrid:

Hyspamérica Ediciones.

Díaz, E. (editora). (1996). La ciencia y el imaginario

social, Buenos Aires: Editorial Biblos.

Díaz, E. (1996), ¿Qué es el imaginario social?

En, E. Díaz (Editora), La ciencia y el imaginario social.

Buenos Aires: Editorial Biblos. 13 - 20

Díaz, E., (1996), La ciencia y el imaginario social.

En, E. Díaz (Editora), La ciencia y el imaginario social.

Buenos Aires: Editorial Biblos. 22 - 27

Díaz, E., (1996), La postsexualidad. El miedo al cuerpo del otro. En, E. Díaz (Editora), La ciencia y el imaginario

social. Buenos Aires: Editorial Biblos. 322 - 327

Feyerabend, P. (1997). Tratado contra el método.

Madrid: Editorial Tecnos.

Flax, J. (1996). La historia de la ciencia: sus motores, sus

frenos, sus cambios, su dirección. En, E. Díaz (Editora),

La ciencia y el imaginario social. Buenos Aires: Editorial

Biblos. $43-55$

Jiménez Vidal, R. (1998) Consideraciones en torno al

pensamiento de Paul Virilio: la deslocalización

cibernética de la comunidad democrática.

Comunicación presentada en el VIII Congreso

Internacional de la AES. 1998. 15-18 de Diciembre de

Granada. Universidad de Sevilla.

Kuhn, T. (1995). La estructura de las revoluciones

científicas. México: Fondo de Cultura Económica. 